\title{
Optimal Mode of clearance in critically ill patients with Acute Kidney Injury (OMAKI) - a pilot randomized controlled trial of hemofiltration versus hemodialysis: a Canadian Critical Care Trials Group project
}

Ron Wald ${ }^{1,2 *}$, Jan O Friedrich ${ }^{2,3,4+}$, Sean M Bagshaw ${ }^{5}$, Karen EA Burns ${ }^{2,3,4}$, Amit X Garg ${ }^{6}$, Michelle A Hladunewich ${ }^{7,8}$, Andrew A House ${ }^{6}$, Stephen Lapinsky ${ }^{4,9}$, David Klein ${ }^{2,3,4,10}$, Neesh I Pannu ${ }^{11}$, Karen Pope ${ }^{10}$, Robert M Richardson ${ }^{12}$, Kevin Thorpe ${ }^{10}$ and Neill KJ Adhikari, ${ }^{4,7 \dagger}$

\begin{abstract}
Introduction: Among critically ill patients with acute kidney injury (AKI) needing continuous renal replacement therapy (CRRT), the effect of convective (via continuous venovenous hemofiltration [CWH]) versus diffusive (via continuous venovenous hemodialysis [CWHD]) solute clearance on clinical outcomes is unclear. Our objective was to evaluate the feasibility of comparing these two modes in a randomized trial.

Methods: This was a multicenter open-label parallel-group pilot randomized trial of CWH versus CWHD. Using concealed allocation, we randomized critically ill adults with AKI and hemodynamic instability to CWH or CWHD, with a prescribed small solute clearance of $35 \mathrm{~mL} / \mathrm{kg} / \mathrm{hour}$ in both arms. The primary outcome was trial feasibility, defined by randomization of $>25 \%$ of eligible patients, delivery of $>75 \%$ of the prescribed CRRT dose, and followup of $>95 \%$ of patients to 60 days. A secondary analysis using a mixed-effects model examined the impact of therapy on illness severity, defined by sequential organ failure assessment (SOFA) score, over the first week.

Results: We randomized 78 patients (mean age 61.5 years; 39\% women; 23\% with chronic kidney disease; 82\% with sepsis). Baseline SOFA scores (mean 15.9, SD 3.2) were similar between groups. We recruited 55\% of eligible patients, delivered $>80 \%$ of the prescribed dose in each arm, and achieved $100 \%$ follow-up. SOFA tended to decline more over the first week in $\mathrm{CWH}$ recipients $(-0.8,95 \% \mathrm{Cl}-2.1,+0.5)$ driven by a reduction in vasopressor requirements. Mortality (54\% CWH; 55\% CWHD) and dialysis dependence in survivors (24\% CWH; 19\% CWHD) at 60 days were similar.
\end{abstract}

Conclusions: Our results suggest that a large trial comparing CWH to CWHD would be feasible. There is a trend toward improved vasopressor requirements among $\mathrm{CWH}$-treated patients over the first week of treatment.

Trial Registration: ClinicalTrials.gov: NCT00675818

\section{Introduction}

Acute kidney injury (AKI) is a common complication of critical illness, afflicting up to two-thirds of patients admitted to the ICU [1]. A significant minority of patients

\footnotetext{
* Correspondence: waldr@smh.ca

† Contributed equally

'Division of Nephrology, St. Michael's Hospital, 30 Bond Street, Toronto, ON, M5B 1W8, Canada

Full list of author information is available at the end of the article
}

with AKI requires renal replacement therapy (RRT), and these individuals have high short-term mortality that ranges from 50 to $70 \%$ [2]. In an attempt to mitigate these poor outcomes, various components of the RRT prescription have been rigorously examined in large well-designed randomized controlled trials (RCTs) [3-5].

The optimal mode of clearance in patients with AKI who require renal support is an area of considerable controversy resulting in significant practice variation [6].

\section{C) Biomed Central}


Hemofiltration, whereby solutes are removed by convection, facilitates the removal of both low and higher molecular weight solutes, depending on the pore size of the membrane [7]. Hemodialysis, in which solute removal occurs via diffusion out of the bloodstream into the dialysate down a concentration gradient, removes low molecular weight molecules but provides limited clearance of higher molecular weight substances. When filter characteristics are kept constant, hemofiltration, which more faithfully mimics glomerular filtration, should result in the clearance of larger-sized solutes as compared to hemodialysis [7]. The removal of such solutes, which may include toxic mediators of sepsis and inflammation, provides the theoretical underpinnings for the superiority of hemofiltration as a renal support mode for critically ill patients with AKI.

We conducted a multicenter pilot RCT of hemofiltration vs. hemodialysis in critically ill patients with AKI to determine whether a larger definitive trial based on clinically important endpoints would be feasible. In a secondary analysis, we evaluated whether hemofiltration improved global organ dysfunction.

\section{Materials and methods Study design}

We conducted an unblinded RCT of continuous venovenous hemofiltration $(\mathrm{CVVH})$ vs. continuous venovenous hemodialysis (CVVHD) with concealed allocation (http:// Clinicaltrials.gov registration number NCT00675818). Our reporting follows the updated CONSORT statement [8].

\section{Setting}

Participants were recruited from ICUs at six academic hospitals: Mt. Sinai Hospital (Medical-Surgical ICU), Sunnybrook Health Sciences Centre (Critical Care Unit and Cardiovascular ICU) and St. Michael's Hospital (Medical-Surgical and Cardiovascular ICUs), all in Toronto, Canada; Victoria Hospital (Critical Care Trauma Centre), and University Hospital (Medical/Surgical Intensive Care Unit and Cardiac Surgery Recovery Unit), both in London, Canada, and University of Alberta Hospital (General Systems Intensive Care Unit) in Edmonton, Canada. The Research Ethics Boards of Mt. Sinai Hospital, St. Michael's Hospital, Sunnybrook Health Sciences Centre, London Health Sciences Centre and the University of Alberta approved the protocol. The Applied Health Research Centre at St. Michael's Hospital (Toronto, Ontario, Canada) was the trial coordinating center.

\section{Population}

We enrolled critically ill adults ( $\geq 16$ years of age) with AKI, defined as a serum creatinine increase $\geq 50 \%$ from baseline (defined as the last known pre-morbid serum creatinine or earliest value available from the current admission). At the time of screening, at least one of the following indications for RRT initiation needed to be present: (i) oliguria (defined as urine output $<100 \mathrm{~mL}$ in the preceding 4 hours); (ii) metabolic acidosis (serum bicarbonate $<15 \mathrm{mmol} / \mathrm{L}$ and $\mathrm{pH}<7.25$ ); (iii) refractory hyperkalemia (serum potassium $>6 \mathrm{mmol} / \mathrm{L}$ despite medical efforts at potassium removal); (iv) serum urea $>50$ $\mathrm{mmol} / \mathrm{L}$, or (v) suspected uremic organ involvement (pericarditis, encephalopathy, neuropathy or myopathy). Finally, participants needed to be hemodynamically unstable, defined as Sequential Organ Failure Assessment (SOFA)- Cardiovascular score $\geq 1$ on the day of screening (see Additional file 1 for the modified SOFA score used in this study). This required the patient to have mean arterial pressure $<70 \mathrm{mmHg}$ or receipt of at least one vasopressor or inotrope [9]. Patients were excluded if any one of the following was present: receipt of any RRT within the previous 2 months; presence of an obstructive etiology for AKI; receipt of a kidney transplant within the preceding year; diagnosis of rapidly progressive glomerulonephritis, vasculitis, or acute interstitial nephritis; a clinical indication for intermittent hemodialysis (for example, the presence of a dialyzable toxin); terminal illness with an associated life expectancy less than 2 months; moribund status (life expectancy $<48$ hours as per judgment of physicians involved in the patient's care); prior enrolment in this study; enrolment in a competing ICU interventional study; non-availability of a CRRT machine, or administration of RRT for > 36 hours prior to eligibility assessment.

\section{Patient allocation}

After eligibility was confirmed, we attempted to obtain consent from the patient or if the patient lacked capacity to consent, his/her substitute decision maker (SDM) was approached. A deferred consent option was approved at three sites, which allowed patient enrollment and randomization in the event of patient incapacity and the inability to locate an SDM. Using this mechanism, patients were randomized, and research personnel attempted to locate an SDM every 72 hours to affirm consent for participation. In all cases, when participants regained capacity, they were asked to provide consent if they were initially enrolled using a priori SDM consent or deferred consent; no participant withdrew consent once regaining capacity. Patients were allocated to a study group using sealed, opaque, sequentially numbered envelopes (prepared by the coordinating center) that were opened after consent was obtained [10]. Randomization was stratified by center in random blocks of four, six or eight. The Research Ethics Board at each center approved the study. An independent data and safety monitoring board tracked the trial's conduct. 


\section{Study intervention}

Participants randomized to CVVH were prescribed ultrafiltration with isovolemic replacement solution (evenly split between pre- and post-filter) to achieve a target clearance of $35 \mathrm{~mL} / \mathrm{kg}$ body weight $/ \mathrm{hr}$. The prescribed hourly ultrafiltration rate was increased above $35 \mathrm{~mL} / \mathrm{kg} / \mathrm{hr}$ to compensate for the reduced efficiency of clearance related to the pre-filter component of the replacement solution volume administered. This adjusted CVVH dose was calculated from the post-filter replacement fluid (RF), pre-filter replacement fluid and blood (blood) flow rates as follows:

Dose $=$ Postfilter RF rate $+(($ Prefilter RF rate $\times($ Blood flow/(Blood flow + Prefilter RF rate))).

In the CVVHD arm, the dialysate flow was set to achieve a clearance of $35 \mathrm{~mL} / \mathrm{kg} / \mathrm{hr}$, which included a post-filter hemofiltration flow of 100 to $200 \mathrm{~mL} / \mathrm{hr}$. This obligate low-volume post-filter hemofiltration is utilized at participating centers to minimize the risk of blood clotting in the machine's deaeration chamber. Ultrafiltration performed for achievement of net fluid balance or to compensate for the volume of administered anticoagulants (namely, citrate or unfractionated heparin) was not considered in the total dose. We used the patient's most recent measured body weight, as recorded in the chart or estimated by the study coordinator if no value was documented, to calculate prescribed RRT dose. Due to difficulties in achieving the target RRT dose as a result of high transmembrane pressures in some patients with higher body mass (particularly those randomized to CVVH), the protocol was modified in May 2010 (21 months after randomization of the first patient) such that the total fluid dose was capped at $4,000 \mathrm{~mL} / \mathrm{hr}$ for both treatment arms, irrespective of the patient's weight. After this protocol change, twenty patients were enrolled in the trial; the $4,000 \mathrm{~mL} / \mathrm{hr}$ dosing cap was invoked in six participants (five allocated to CVVHD and one to CVVH) who would have otherwise needed higher flows to achieve an actual total dose of $35 \mathrm{~mL} / \mathrm{kg} / \mathrm{hr}$.

All study therapies were delivered by the Gambro Prismaflex ${ }^{\mathrm{TM}}$ RRT machine using the ST100 (surface area $1.0 \mathrm{~m}^{2}$ ) or ST150 (surface area $1.5 \mathrm{~m}^{2}$ ) filter sets, which contain a polyacrylonitrile AN69 membrane (Gambro, Richmond Hill, ON, Canada). We permitted the use of any commercially available dialysate and replacement solutions. Decisions regarding circuit anticoagulation (heparin, regional citrate anticoagulation, or no anticoagulation) and volume control were at the discretion of the attending physicians. Patients remained on study therapy until death, withdrawal of CRRT as part of withdrawal of life support, hemodynamic stability (SOFA-cardiovascular score $<2$ for $>24$ hrs) permitting stepdown to intermittent hemodialysis, or recovery of kidney function (defined as urine output $>500 \mathrm{~mL}$ in the preceding $12 \mathrm{hrs}$, and most recent serum potassium $<5.5 \mathrm{mmol} / \mathrm{L}$ and serum bicarbonate $>18 \mathrm{mmol} / \mathrm{L}$ ).

\section{Outcomes}

The primary feasibility outcome of this study was the ability to administer $>75 \%$ of the prescribed CRRT dose to participants in each treatment arm. Secondary feasibility outcomes included the ability to enroll $>25 \%$ of fully eligible patients and the ability to follow $>95 \%$ of patients to 60 days following randomization (the anticipated follow-up period for the future definitive principal study). Secondary outcomes included change in SOFA score from baseline to days 1, 2 and 7, respectively, following randomization. Serial changes in SOFA scores have been shown to be correlated with clinical outcomes in critically ill patients with AKI who require RRT [11].

\section{Data collection}

Trained research coordinators collected baseline clinical and demographic data, and information on pre-existing medical conditions. Specific risk factors for AKI were ascertained, including recent procedures, nephrotoxins, and sepsis (defined using consensus guidelines [12]). SOFA score was calculated at the time of randomization and on each day of study therapy. The SOFA-Cardiovascular score was modified to include the receipt of vasopressin. Patients receiving RRT on a given day were assigned a SOFA-Renal score of 4, regardless of urine output or serum creatinine. Participants were followed until death or a maximum of 60 days from randomization, at which time vital status and the ongoing need for RRT among survivors were recorded.

\section{Statistical analyses}

As this was a feasibility trial with the primary objective of informing the design of a large-scale RCT, we planned to enroll a convenience sample of 75 participants from six sites. Patients who were randomized but never received RRT are described, but these individuals were replaced to ensure that at least 75 patients received some form of RRT. Since the primary feasibility outcome was based on the dose of CRRT received, patients for whom dose could not be readily calculated (those who received no RRT or forms of RRT other than CRRT) were excluded from the analysis related to feasibility. However, clinical outcomes are reported for all randomized participants.

Descriptive statistics were used to characterize participants in either arm. Continuous variables are presented as means (SD) or medians (interquartile range, IQR) and two-group comparisons were performed with the $t$-test or Wilcoxon test, as appropriate. Two-group comparisons involving categorical variables were carried out with the chi square test. Analysis of covariance, adjusted for baseline SOFA score, was used to evaluate the change in 
SOFA score on days 1 and 2. Linear mixed models adjusted for baseline SOFA score and day of study therapy were used to evaluate the impact of RRT mode on SOFA score over the first week of therapy. For the fixed effect of treatment (that is, CVVH vs. CVVHD) 95\% confidence intervals $(\mathrm{CI})$ were obtained by profiling the log-likelihood function. All analyses were performed using R version 2.12.0 (R Development Core Team 2010, Vienna, Austria).

\section{Results}

We screened 347 patients; 143 were eligible for participation and 79 individuals (55.2\%) were enrolled over a 24-month time period. The inability to obtain consent from the patient or SDM was the reason for the nonenrollment of otherwise eligible patients. One patient was excluded shortly after enrollment after it was decided to pursue a non-continuous form of RRT. In total, 78 patients were randomized (39 to $\mathrm{CVVH}$, and 39 to CVVHD). In one case, prior to the start of therapy it was recognized that a patient randomized to CVVHD was inappropriately enrolled as the indication for RRT was toxin removal rather than AKI per se. This patient was excluded from all further analyses. Clinical outcomes are reported in an intention-to-treat fashion for the remaining 77 patients (CVVH, 39; CVVHD, 38). Four patients randomized to $\mathrm{CVVH}$ were excluded from the feasibility analysis, two due to death prior to commencement of study RRT, and two due to receipt of continuous venovenous hemodiafiltration as the initial mode of therapy. The indication(s) for RRT was (were) oliguria, metabolic acidosis, hyperkalemia and uremia in 34, 17, 6 and 8 patients, respectively, in the CVVH arm. In the CVVHD arm, these indications guided the inclusion of 36, 15, 4 and 3 participants, respectively. In total, 73 participants commenced the therapy to which they were randomized (CVVH, 35; CVVHD, 38); these individuals contributed to the analysis relating to the feasibility of treatment delivery (Figure 1).

\section{Baseline characteristics (Table 1)}

The mean age of participants was 61.5 (SD 14.2) years and $30 / 77$ (39\%) were women. The median time from ICU admission to randomization was 2 (IQR 2 to 8 ) days. Sepsis was present in 63/77 (82\%) of participants, $73 / 77$ (95\%) were mechanically ventilated and 69/77 (90\%) received vasopressors at the time of randomization. Median 24-hr urine output was 125 (IQR 50 to 250) $\mathrm{mL}$. Approximately two-thirds of participants received some form of RRT prior to randomization, with 23/39 in the CVVH group (59\%) for a mean of 10.6 (SD 12.0) hrs, and $30 / 38$ in the CVVHD group (79\%) for a mean of 13.3 (SD 11.7) hrs. (Table 1

\section{Features of study treatments (Table 2)}

Among the 35 participants who started CVVH, RRT was prescribed for a median of 107 (IQR 55 to 146) hrs and delivered for 85 (IQR 43 to 128) hrs. Overall, 84.7\% (95\%

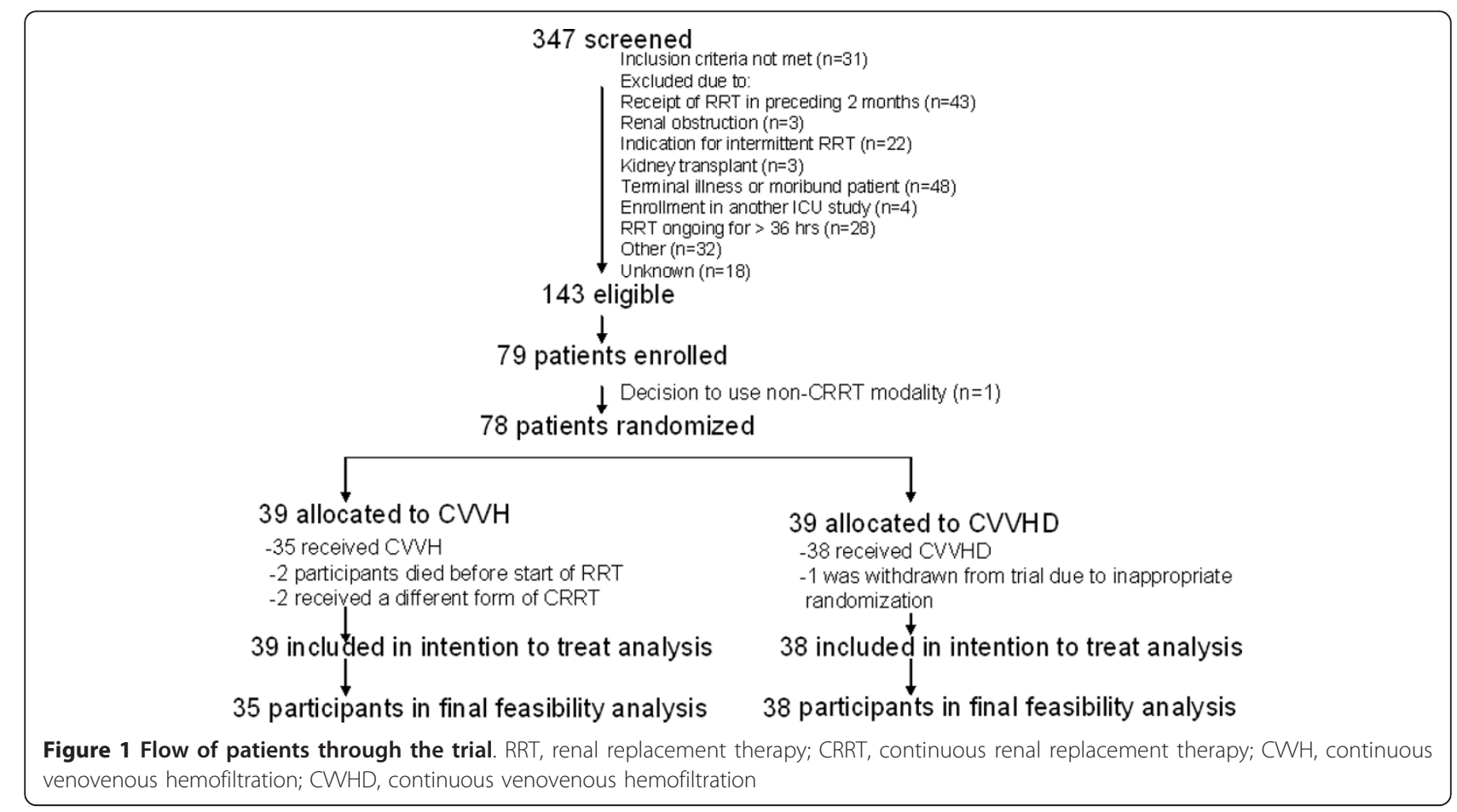


Table 1 Baseline characteristics

\begin{tabular}{|c|c|c|}
\hline & CVVH $(n=39)$ & CVVHD $(n=38)$ \\
\hline Age, years & $58.8 \pm 13.7$ & $64.3 \pm 14.3$ \\
\hline Female & $14(36 \%)$ & $16(42 \%)$ \\
\hline Weight, kg & $86.6 \pm 31.5$ & $89.2 \pm 26.4$ \\
\hline Days from ICU admission to randomization & $2(1,4)$ & $2(1,3)$ \\
\hline SOFA score & $16.3 \pm 3.3$ & $15.5 \pm 3.0$ \\
\hline \multicolumn{3}{|l|}{ Premorbid conditions } \\
\hline Hypertension & $19(49 \%)$ & $26(68 \%)$ \\
\hline Diabetes mellitus & $9(23 \%)$ & $9(24 \%)$ \\
\hline Chronic kidney disease & $5(13 \%)$ & $13(34 \%)$ \\
\hline \multicolumn{3}{|l|}{ Acute kidney injury risk factors } \\
\hline Sepsis & $31(79 \%)$ & $32(84 \%)$ \\
\hline Cardiopulmonary bypass in past 7 days & $1(3 \%)$ & $4(11 \%)$ \\
\hline IV contrast in past 7 days & $9(23 \%)$ & $6(16 \%)$ \\
\hline \multicolumn{3}{|l|}{ Physiologic parameters } \\
\hline Urine output, mL/24 hr & $125(60,245)$ & $135(46,251)$ \\
\hline Minimum systolic BP during $24 \mathrm{hr}$ before enrolment, mmHg & $94.2 \pm 16.5$ & $86.1 \pm 13.5$ \\
\hline Minimum diastolic BP during $24 \mathrm{hr}$ before enrolment, $\mathrm{mmHg}$ & $48.9 \pm 8.6$ & $47.2 \pm 9.4$ \\
\hline \multicolumn{3}{|l|}{ Laboratory parameters } \\
\hline Serum creatinine, $\mu \mathrm{mol} / \mathrm{L}$ & $276(194,352)$ & $246(155,325)$ \\
\hline Urea, mmol/L & $25.8 \pm 38.1$ & $17.6 \pm 12.7$ \\
\hline Potassium, mmol/L & $4.3 \pm 0.7$ & $4.1 \pm 0.6$ \\
\hline Bicarbonate, $\mathrm{mmol} / \mathrm{L}$ & $20.5 \pm 5.9$ & $20.2 \pm 5.3$ \\
\hline Hemoglobin, $g / L$ & $86.1 \pm 16.3$ & $90.0 \pm 15.2$ \\
\hline Platelets, $\times 10^{9} / \mathrm{L}$ & $109.6 \pm 82.6$ & $127.2 \pm 80.9$ \\
\hline $\mathrm{WBC}, \times 10^{9} / \mathrm{L}$ & $20.2 \pm 43.3$ & $16.9 \pm 10.6$ \\
\hline \multicolumn{3}{|l|}{ ICU interventions } \\
\hline Duration of RRT prior to randomization, hours & $8(0,19)$ & $13(1.0,20.5)$ \\
\hline Mechanical ventilation & $37(95 \%)$ & $36(95 \%)$ \\
\hline Vasopressors & $34(87 \%)$ & $35(92 \%)$ \\
\hline Total parenteral nutrition & $1(3 \%)$ & $2(5 \%)$ \\
\hline
\end{tabular}

Continuous data are displayed as mean \pm SD or medians (interquartile range), as appropriate. Categorical variables are displayed as $\mathrm{n}$ (\%). CRRT, continuous renal replacement therapy; CVVH, continuous venovenous hemofiltration; CVVHD, continuous venovenous hemodialysis; BP, blood pressure; RRT, renal replacement therapy; SOFA, Sequential Organ Failure Assessment; WBC, White blood cell.

CI 79.1, 90.3) of the prescribed duration was delivered. The mean weight-standardized dose was 33.6 (SD 7.4) $\mathrm{mL} / \mathrm{kg} / \mathrm{hr}$ while CRRT was ongoing.(Table 2

CVVHD was prescribed in 38 participants for a median of 92 (IQR 57 to 145) hrs and delivered for 76 (IQR 44 to 148 ) hrs. Over the course of the trial, $87.8 \%$ (95\% CI $83.3,92.3)$ of the prescribed duration was delivered to patients randomized to receive CVVHD. The mean weight-standardized delivered RRT dose was $34.7 \mathrm{~mL} / \mathrm{kg} /$ hr (SD 4.4).

\section{Clinical outcomes}

All subjects were followed to 60 days, by which point 22/ $39(56 \%)$ and $21 / 38$ (55\%) of participants assigned to CVVH and CVVHD, respectively, had died. Among surviving patients, 4/17 (24\%) and 3/17 (19\%) of those initially assigned to CVVH and CVVHD respectively, were still dependent on RRT.
After adjustment for baseline SOFA score, we found a non-significant decline in SOFA score among participants treated with CVVH compared to CVVHD on the first day $(-0.4,95 \%$ CI $-1.3,0.6)$ and second day $(-0.4,95 \%$ CI -1.6 , 0.8 ) following randomization. Over the first week of therapy, the adjusted change in the SOFA score among participants treated with CVVH compared to CVVHD was -0.8 (95\% CI -2.1, 0.5). The observed reduction appeared to be driven by a reduction in the cardiovascular component of the SOFA score (Figure 2).

\section{Protocol violations and adverse events}

We identified 15 protocol violations involving 15 participants. In one individual who started therapy with $\mathrm{CVVH}$, achievement of the target dose was not feasible and RRT was supplemented with the addition of continuous dialysis; ongoing difficulties with maintaining adequate small molecule clearance using CRRT resulted in a change to 
Table 2 Feasibility and safety data

\begin{tabular}{|c|c|c|c|}
\hline & $\begin{array}{l}\text { CVVH } \\
(n=35)\end{array}$ & $\begin{array}{l}\text { CVVHD } \\
(n=38)\end{array}$ & $P$-value \\
\hline Duration RRT prescribed, hrs & $146 \pm 240$ & $145 \pm 156$ & 0.88 \\
\hline Duration RRT received, hrs & $130 \pm 222$ & $128 \pm 142$ & 0.87 \\
\hline Mean pre-filter replacement solution flow, $\mathrm{mL} / \mathrm{hr}$ & $1533 \pm 442$ & 0 & $\mathrm{n} / \mathrm{a}$ \\
\hline Mean post-filter replacement solution flow, $\mathrm{mL} / \mathrm{hr}$ & $1440 \pm 488$ & $180 \pm 140$ & $\mathrm{n} / \mathrm{a}$ \\
\hline Mean dialysate flow, $\mathrm{mL} / \mathrm{hr}$ & 0 & $2871 \pm 872$ & $\mathrm{n} / \mathrm{a}$ \\
\hline Mean RRT dose, mL/kg/hr & $33.6 \pm 7.4$ & $34.7 \pm 4.4$ & 0.50 \\
\hline Prescribed dose delivered, $\%$ & $84.7 \pm 16.3$ & $87.8 \pm 13.7$ & 0.73 \\
\hline Net ultrafiltration, L/day & $1.7 \pm 2.2$ & $0.8 \pm 4.1$ & 0.98 \\
\hline Days on study therapy & $5(3-7)$ & $4.50(3.00-10.25)$ & 0.79 \\
\hline Primary reason for CRRT withdrawal & & & 0.75 \\
\hline Death while on CRRT & $12(35 \%)$ & $10(27 \%)$ & \\
\hline Kidney function recovery & $7(21 \%)$ & $7(19 \%)$ & \\
\hline Transfer to intermittent hemodialysis & $12(35 \%)$ & $14(38 \%)$ & \\
\hline Withdrawal of life support & $3(9 \%)$ & $6(16 \%)$ & \\
\hline Catheter changes/day of therapy & $0.09 \pm 0.2$ & $0.1 \pm 0.2$ & 0.92 \\
\hline Units RBCs transfused/day of therapy & $0.3 \pm 0.3$ & $0.4 \pm 0.7$ & 0.38 \\
\hline Unscheduled circuit changes/day of therapy & $0.2 \pm 0.3$ & $0.2 \pm 0.2$ & 0.36 \\
\hline Study days with receipt of norepinephrine, \% & $61.8 \pm 36.2$ & $65.7 \pm 7.5$ & 0.41 \\
\hline Study days with receipt of vasopressin, $\%$ & $35.4 \pm 35.9$ & $43.0 \pm 43.6$ & 0.51 \\
\hline
\end{tabular}

Continuous data are displayed as mean \pm standard deviation or median (interquartile range), as appropriate. Categorical variables are displayed as $\mathrm{n}$ (\%); $\mathrm{n} / \mathrm{a}, \mathrm{not}$ applicable. Data are restricted to patients who initiated therapy with the CRRT mode to which they had been allocated. RRT, renal replacement therapy; CRRT, continuous renal replacement therapy; RBCs, red blood cells; $\mathrm{CVVH}$, continuous venovenous hemofiltration; CVVHD, continuous venovenous hemofiltration.

sustained low efficiency dialysis. In five cases (one patient on CVVH, and four on CVVHD), stepdown from CRRT to IHD occurred before the participant met the studydefined criteria for hemodynamic stability. In five cases (two patients on CVVH, and three on CVVHD), RRT was discontinued altogether in participants who did not meet study criteria for renal recovery.
No adverse events were attributed to the study intervention in either treatment arm.

\section{Discussion}

We completed a multi-center, concealed-allocation, randomized trial comparing hemofiltration and hemodialysis in critically ill patients with AKI. This pilot trial achieved

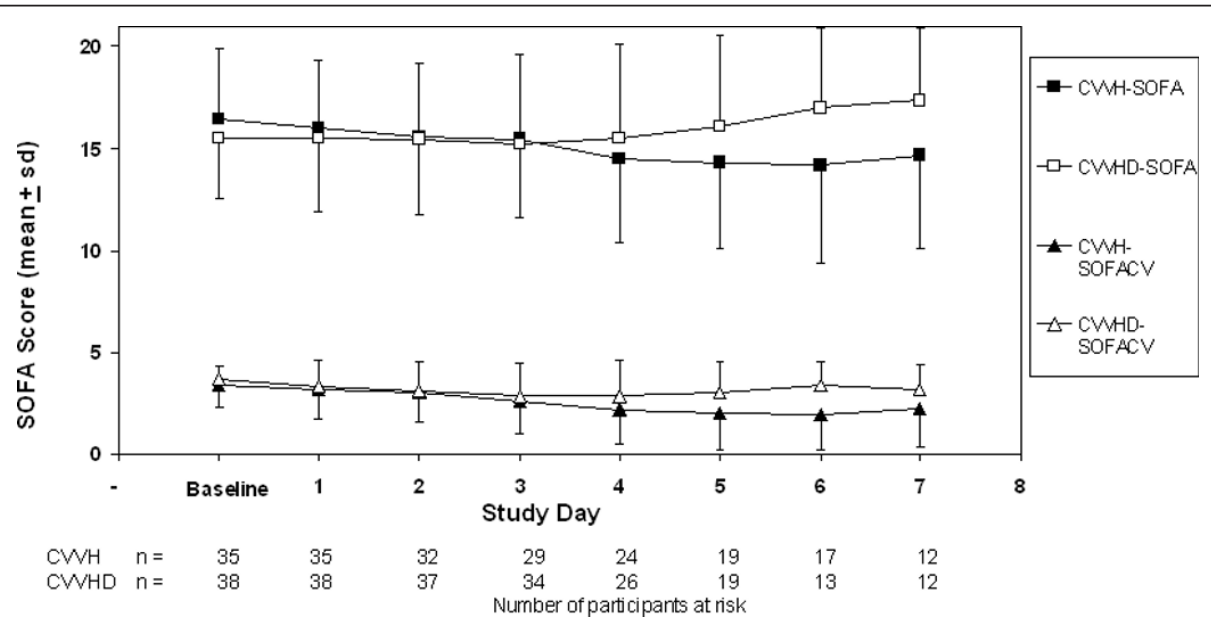

Figure 2 Total and Cardiovascular (CV) Sequential Organ Failure Assessment (SOFA) scores over the first seven days following randomization. Total SOFA scores are denoted with squares; the cardiovascular component of the SOFA score is denoted with triangles.CWH, continuous venovenous hemofiltration; CWHD, continuous venovenous hemofiltration 
its primary objective of confirming the feasibility of performing a large-scale trial evaluating RRT clearance mode in AKI. There was also a non-significant trend towards reduced organ dysfunction, driven by decreased vasopressor requirements, early after the initiation of RRT in patients who received hemofiltration. These feasibility data and the importance of the clinical question justify the conduct of a large trial with adequate power to evaluate the primary outcome of 60 -day mortality. With a power of 0.80 , type I error of 0.05 , and a mortality reduction of $10 \%$ in patients treated with CVVH (estimated 60-day mortality in the CVVHD arm 55\%), we estimate that such a trial would require the enrolment of nearly 400 patients per arm. A more conservative mortality reduction of $7.5 \%$ would require the enrolment of 700 patients per arm.

Little is known about the optimal mode of clearance in renal replacement for AKI. Although both convection and diffusion remove small molecules with equal efficiency, convection may remove larger molecules that are not cleared by diffusive mechanisms [13]; previous studies have examined effects on inflammatory markers and have been too small to reliably determine effects on clinical outcomes. In addition, the profile of larger molecules removed by convection is relatively non-specific and may also include molecules that dampen inflammation or crucial medications such as antibiotics [14]. A randomized crossover study of 13 patients with AKI and the systemic inflammatory response syndrome found that $\mathrm{CVVH}$ for $24 \mathrm{hrs}$ reduced plasma concentrations of TNF $\alpha$ and cleared more IL-6, compared to CVVHD. However, there was no effect on plasma concentrations of IL-6, IL-10, SLselectin, or endotoxin [15]. Morgera and coworkers randomized 24 patients with sepsis-associated AKI to treatment with CVVH or CVVHD using a high cutoff membrane permeable to molecules up to 60 kilodaltons in size. Plasma concentration and clearance for IL-6 did not differ, but clearance of IL-1 receptor antagonist, an anti-inflammatory mediator, was enhanced by CVVH [16]. Of note, protein losses were higher in patients who received $\mathrm{CVVH}$. In a prospective crossover study involving 15 patients with AKI who sequentially received $\mathrm{CVVH}$ and CVVHD, $\beta_{2}$-microglobulin clearance was non-significantly higher among $C V V H$ recipients $(P=0.055)$ [17]. Among trials focusing on clinical outcomes, a single-centre RCT $(\mathrm{n}=20)$ did not demonstrate a difference in survival, renal recovery or ICU stay in patients treated with CVVH vs. CVVHD administered at fixed doses of 1.7 to $2.0 \mathrm{~L} / \mathrm{hr}$ [18]. Similarly, a recent unpublished 65-patient RCT of CVVH vs continuous venovenous hemodiafiltration (both at $40 \mathrm{~mL} / \mathrm{kg} / \mathrm{hr}$ ) did not demonstrate a survival difference at 28 days [19].

While interventional trials involving devices and processes of care that are susceptible to large variations in practice are challenging, we achieved our feasibility objectives. Specifically, we were able to recruit the majority of eligible subjects, implement the protocolized therapy for $>85 \%$ of the prescribed time and ascertain vital status at 60 days for all participants. When accounting for actual time on therapy, the delivered dose exceeded $80 \%$ of that prescribed in both treatment arms, thereby surpassing our feasibility threshold of $75 \%$. Accordingly, we believe our study strongly supports the feasibility of a large definitive randomized trial comparing hemofiltration and hemodialysis in critically ill patients with severe AKI.

This is the largest published trial to date to study the mode of solute clearance in AKI. Given the challenges of recruiting participants and implementing interventions in a population with a high burden of illness, the success of our pilot was a necessary precursor to a principal trial that examines patient-relevant clinical outcomes. Our eligibility criteria were pragmatic and assured the inclusion of individuals in North America who typically receive CRRT. A minority of individuals for whom no SDM could be found were enroled with deferred consent, thereby limiting exclusion of potentially eligible patients and mitigating selection bias. Of interest, no subject enroled by deferred consent had an SDM who subsequently withdrew consent or withdrew consent personally after regaining capacity. Finally, other than the clearance mode, all other aspects of RRT including stepdown to intermittent hemodialysis and withdrawal of RRT were performed in a manner consistent with usual practice.

Our study has several limitations. As this was an unblinded trial, we cannot exclude the effect of co-interventions in either treatment arm. However, the nature of our intervention made blinding impractical, and we ensured that the two groups received equivalent RRT doses. There is also no definite intervention related to RRT prescription that has been shown to modify outcomes in critically ill patients with AKI. In addition, we cannot exclude the possibility that patients who were eligible but not randomized were systematically different than trial participants. Our protocol specified a target clearance of $35 \mathrm{~mL} / \mathrm{kg} / \mathrm{hr}$, which was generally achieved in both arms. The decision to use this dose was guided by the fact that our trial was designed when higher dose CRRT was felt to be potentially beneficial based on data from two trials $[20,21]$. This pre-dated more recent trials and metaanalyses demonstrating no advantage of higher dose CRRT over doses of 20 to $25 \mathrm{~mL} / \mathrm{kg} / \mathrm{hr}$, which would likely be the standard in a future trial comparing convection and diffusion $[4,5,22,23]$. However, since high dose therapy appears safe and may positively modify the effect of convective clearance [20], our higher dose target was reasonable. It should be noted that while $35 \mathrm{ml} / \mathrm{kg} / \mathrm{hr}$ was prescribed to all participants, actual solute clearance may 
have been significantly lower due to changes in filter permeability $[24,25]$. On the other hand, when calculating the prescribed dose, we did not consider clearance associated with net ultrafiltration or removal of volume associated with the administration of anticoagulants. This approach would tend to underestimate the actual solute clearance that was delivered. Importantly, any deviation in dose from our $35 \mathrm{~mL} / \mathrm{kg} / \mathrm{hr}$ target would affect participants in both intervention arms to a similar extent. The Prismaflex CRRT system requires that all patients (even those predominantly getting hemodialysis) receive a small amount of post-filter hemofiltration (up to $200 \mathrm{ml} / \mathrm{hr}$ ) to prevent clotting in the machine's deaeration chamber. In addition, CVVHD recipients would have had other unavoidable sources of convective clearance, specifically for the achievement of net fluid removal, and for the isovolemic removal of volume associated with the administration of the anticoagulant (citrate or heparin). Thus, CVVHD recipients did not receive purely diffusive solute clearance. We nonetheless estimate that the typical patient enrolled in the CVVHD arm still received $>80 \%$ of therapy in the form of diffusive clearance, which reassures us that this trial truly compared two different modes of solute clearance. Our trial was conducted using the AN69 polyacryonitrile filter, which has unique adsorptive characteristics [14]. While this is a widely used membrane in the administration of CRRT, the adsorptive characteristics of filters may differ and we cannot generalize our findings to settings in which other filters are utilized. Moreover, we did not collect data to evaluate the relative effect of CVVH or CVVHD on the removal of molecules of varying size. This trial was conducted using continuous RRT. Although the question of hemodialysis vs. hemofiltration is applicable to patients stable enough to receive intermittent RRT, machines to deliver intermittent hemofiltration were not widely available in North America when the trial was conducted. Among trial participants who became more hemodynamically stable, intermittent hemodialysis was utilized even when the initial CRRT mode was hemofiltration, thereby potentially diluting the benefits associated with CVVH. Finally, while we observed a trend towards improved organ failure scores, this finding must be interpreted with caution given the small sample size of our trial. Moreover, disease severity scores such as the SOFA score are surrogate markers that cannot supplant hard clinical endpoints.

\section{Conclusions}

Our findings clearly support the feasibility of performing a definitive trial comparing CVVH and CVVHD in critically ill patients with AKI. The early non-significant trend towards reduced vasopressor requirements provides preliminary support to the concept that convective modes of clearance reduce inflammation and thus benefit critically ill patients. Given the high mortality associated with AKI, the lack of specific RRT interventions shown to reduce mortality, and current practice variation, the results of our pilot trial provide justification for a larger trial of hemofiltration vs. hemodialysis that will be adequately powered to evaluate meaningful clinical outcomes.

\section{Key messages}

- A randomized controlled trial of hemofiltration vs hemodialysis is feasible.

- Hemofiltration may be associated with decreased vasopressor requirements over the first week of therapy.

- A well-designed and adequately powered trial of hemofiltration vs hemodialysis would address an important area of uncertainty in the management of patients with AKI.

\section{Additional material}

Additional file 1: Modified Sequential Organ Failure Assessment

(SOFA) score. SOFA score modified from the original version (see reference [9]) for application in the OMAKI trial.

\section{Abbreviations}

AKI: acute kidney injury; CRRT: continuous renal replacement therapy; $\mathrm{CWH}$ : continuous venovenous hemofiltration; CWHD: continuous venovenous hemodialysis; IL: interleukin; IQR: interquartile range; RF: replacement fluid; RCT: randomized controlled trial; RRT: renal replacement therapy; SDM: substitute decision maker; SOFA: Sequential Organ Failure Assessment; TNF a: tumor necrosis factor alpha.

\section{Acknowledgements}

The investigators thank the members of the Data Safety Monitoring Board (Orfeas Liangos, MD (Chair), Vanita Jassal, MD, and Damon Scales, MD, PhD) for their time. We appreciate the hard work of the site coordinators (Nicole Marinoff, Derek Parsotam, Orla Smith, Kerri Porretta, Yoon Lee, Virginia Schumann, Samuel Vijayan, Laura Webster, Steve Ramganesh, Karen O-HaraBanack, and Samantha Taylor). Central coordination of the trial was conducted at the Applied Health Research Centre at St. Michael's Hospital, Toronto, ON and we appreciate the efforts of Dr. Muhammad Mamdani, Director, and staff members who made this trial possible (Judith Hall, Nike Onabajo, Ping Wee, and Vicky Vuong).

This study was supported by the Canadian Institutes of Health Research (CIHR) RCT Mentoring Program (to Dr. Wald), by an unrestricted grant from the Baxter-Oreopoulos Fund, Division of Nephrology, University of Toronto, and by the Academic Enrichment Fund, Department of Critical Care Medicine, Sunnybrook Health Sciences Centre. Drs Friedrich and Garg are supported by a CIHR Clinician-Scientist award. Dr Bagshaw is supported by a Canada Research Chair in Critical Care Nephrology and Clinical Investigator award from Alberta Innovates - Health Solutions.

\section{Author details}

'Division of Nephrology, St. Michael's Hospital, 30 Bond Street, Toronto, ON M5B 1W8, Canada. ${ }^{2}$ Keenan Research Centre in the Li Ka Shing Knowledge Institute of St. Michael's Hospital, 30 Bond Street, Toronto, ON, M5B 1W8, Canada. ${ }^{3}$ Critical Care and Medicine Departments, St. Michael's Hospital, 30 Bond Street, Toronto, ON, M5B 1W8, Canada. ${ }^{4}$ Interdepartmental Division of Critical Care, University of Toronto, 30 Bond Street, Toronto, ON, M5B 1W8, Canada. ${ }^{5}$ Division of Critical Care Medicine, University of Alberta, 8440112 Street NW. Edmonton, AB, T6G 2B7, Canada. 'Division of Nephrology, London Health Sciences Centre, 800 Commissioners Road East, London, ON, 
N6A 5W9, Canada. 'Division of Nephrology, Sunnybrook Health Sciences Centre, 2075 Bayview Avenue, Toronto, ON, M4N 3M5, Canada. ${ }^{8}$ Department of Critical Care Medicine and Sunnybrook Research Institute, Sunnybrook Health Sciences Centre, 2075 Bayview Avenue, Toronto, ON, M4N 3M5, Canada. ${ }^{9}$ Division of Critical Care, Mt. Sinai Hospital, 600 University Avenue, Toronto, ON, M5G 1X5, Canada. ${ }^{10}$ Applied Health Research Centre, Li Ka Shing Knowledge Institute, St. Michael's Hospital, 30 Bond Street, Toronto, ON, M5B 1W8, Canada. " ${ }^{11}$ Division of Nephrology, University of Alberta Hospital, 8440112 Street NW, Edmonton, Alberta, T6G 2B7, Canada. ${ }^{12}$ Division of Nephrology, University Health Network, 200 Elizabeth Street, Toronto, ON, M5G 2C4, Canada.

\section{Authors' contributions}

RW conceived and designed the study, obtained funding, supervised study conduct, interpreted the data, drafted the manuscript, and provided critical review of the manuscript. JOF conceived and designed the study, supervised study conduct, interpreted the data, drafted the initial version of the manuscript, and provided critical review of the manuscript. SMB interpreted the study data, supervised study conduct, and provided critical review of the manuscript. KEB interpreted the study data, supervised study conduct, and provided critical review of the manuscript. AXG interpreted the study data, supervised study conduct, and provided critical review of the manuscript. MAH interpreted the study data, supervised study conduct, and provided critical review of the manuscript. AAH interpreted the study data, supervised study conduct, and provided critical review of the manuscript. SL interpreted the study data, supervised study conduct and provided critical review of the manuscript. DK interpreted the study data, supervised study conduct, and provided critical review of the manuscript. RMR interpreted the study data, supervised study conduct, and provided critical review of the manuscript. NIP interpreted the study data and provided critical review of the manuscript. KP participated in data analysis and interpretation, designed data collection tools, and participated in the critical review of the manuscript. KT participated in study design, conducted the data analysis, participated in data interpretation, and in the critical review of the manuscript. NKJA conceived and designed the study, supervised study conduct, interpreted the data, drafted the initial version of the manuscript, and provided critical review of the manuscript. All authors approved the final version.

\section{Competing interests}

Dr. Bagshaw has served on a Gambro Experts Panel. No other relevant competing interests are reported.

Received: 10 May 2012 Revised: 1 October 2012

Accepted: 3 October 2012 Published: 24 October 2012

\section{References}

1. Hoste EA, Schurgers M: Epidemiology of acute kidney injury: how big is the problem? Crit Care Med 2008, 36:S146-151.

2. Uchino S, Kellum JA, Bellomo R, Doig GS, Morimatsu H, Morgera S, Schetz M, Tan I, Bouman C, Macedo E, Gibney N, Tolwani A, Ronco C: Acute renal failure in critically ill patients: a multinational, multicenter study. Jama 2005, 294:813-818

3. Vinsonneau C, Camus C, Combes A, Costa de Beauregard MA, Klouche K, Boulain T, Pallot JL, Chiche JD, Taupin P, Landais P, Dhainaut JF: Continuous venovenous haemodiafiltration versus intermittent haemodialysis for acute renal failure in patients with multiple-organ dysfunction syndrome: a multicentre randomised trial. Lancet 2006, 368:379-385.

4. Palevsky PM, Zhang JH, O'Connor TZ, Chertow GM, Crowley ST, Choudhury D, Finkel K, Kellum JA, Paganini E, Schein RM, Smith MW, Swanson KM, Thompson BT, Vijayan A, Watnick S, Star RA, Peduzzi P: Intensity of renal support in critically ill patients with acute kidney injury. N Engl I Med 2008, 359:7-20.

5. Bellomo R, Cass A, Cole L, Finfer S, Gallagher M, Lo S, McArthur C, McGuinness S, Myburgh J, Norton R, Scheinkestel C, Su S: Intensity of continuous renal-replacement therapy in critically ill patients. N Engl J Med 2009, 361:1627-1638.

6. Ricci Z, Ronco C, D'Amico G, De Felice R, Rossi S, Bolgan I, Bonello M, Zamperetti N, Petras D, Salvatori G, Dan M, Piccinni P: Practice patterns in the management of acute renal failure in the critically ill patient: an international survey. Nephrol Dial Transplant 2006, 21:690-696.
7. Depner TA: "Artificial" hemodialysis versus "natural" hemofiltration. Am J Kidney Dis 2008, 52:403-406.

8. Schulz KF, Altman DG, Moher D: CONSORT 2010 statement: updated guidelines for reporting parallel group randomized trials. Ann Intern Med 152:726-732.

9. Ferreira FL, Bota DP, Bross A, Melot C, Vincent JL: Serial evaluation of the SOFA score to predict outcome in critically ill patients. JAMA 2001, 286:1754-1758.

10. Doig GS, Simpson F: Randomization and allocation concealment: a practical guide for researchers. J Crit Care 2005, 20:187-191, discussion 191-183.

11. Cappi SB, Sakr Y, Vincent JL: Daily evaluation of organ function during renal replacement therapy in intensive care unit patients with acute renal failure. J Crit Care 2006, 21:179-183.

12. Bone RC, Balk RA, Cerra FB, Dellinger RP, Fein AM, Knaus WA, Schein RM, Sibbald WJ: Definitions for sepsis and organ failure and guidelines for the use of innovative therapies in sepsis. The ACCP/SCCM Consensus Conference Committee. American College of Chest Physicians/Society of Critical Care Medicine. Chest 1992, 101:1644-1655.

13. Brunet S, Leblanc M, Geadah D, Parent D, Courteau S, Cardinal J: Diffusive and convective solute clearances during continuous renal replacement therapy at various dialysate and ultrafiltration flow rates. Am J Kidney Dis 1999, 34:486-492.

14. De Vriese AS, Colardyn FA, Philippe JJ, Vanholder RC, De Sutter JH, Lameire NH: Cytokine removal during continuous hemofiltration in septic patients. J Am Soc Nephrol 1999, 10:846-853.

15. Kellum JA, Johnson JP, Kramer D, Palevsky P, Brady JJ, Pinsky MR: Diffusive vs. convective therapy: effects on mediators of inflammation in patient with severe systemic inflammatory response syndrome. Crit Care Med 1998, 26:1995-2000.

16. Morgera S, Slowinski T, Melzer C, Sobottke V, Vargas-Hein O, Volk T, Zuckermann-Becker H, Wegner B, Muller JM, Baumann G, Kox WJ, Bellomo R, Neumayer HH: Renal replacement therapy with high-cutoff hemofilters: Impact of convection and diffusion on cytokine clearances and protein status. Am J Kidney Dis 2004, 43:444-453.

17. Ricci Z, Ronco C, Bachetoni A, D'Amico G, Rossi S, Alessandri E, Rocco M, Pietropaoli P: Solute removal during continuous renal replacement therapy in critically ill patients: convection versus diffusion. Crit Care 2006, 10:R67.

18. Daud KM, Leong G, Visvanathan R: Acute Dialytic Support for the Critically III: Continuous Venovenous Haemodialysis versus Continuous Venovenous Haemofiltration. International Medical Journal 2006, 13:37-42.

19. Chang JW, Lee HK, Wang WS, Kim SB, Park S-K, Lee SK, Park JS: No difference in clearance and survival between continuous hemodiltration and hemodiafiltration at the same net effluent in patients with acute renal failure. Crit Care Med 2009, 37:A473.

20. Ronco C, Bellomo R, Homel P, Brendolan A, Dan M, Piccinni P, La Greca G: Effects of different doses in continuous veno-venous haemofiltration on outcomes of acute renal failure: a prospective randomised trial. Lancet 2000, 356:26-30.

21. Saudan P, Niederberger M, De Seigneux S, Romand J, Pugin J, Perneger T, Martin PY: Adding a dialysis dose to continuous hemofiltration increases survival in patients with acute renal failure. Kidney Int 2006, 70:1312-1317.

22. Jun M, Heerspink HJ, Ninomiya T, Gallagher M, Bellomo R, Myburgh J, Finfer S, Palevsky PM, Kellum JA, Perkovic V, Cass A: Intensities of renal replacement therapy in acute kidney injury: a systematic review and meta-analysis. Clin J Am Soc Nephrol 2010, 5:956-963.

23. Van Wert R, Friedrich JO, Scales DC, Wald R, Adhikari NK: High-dose renal replacement therapy for acute kidney injury: Systematic review and meta-analysis. Crit Care Med 2010, 38:1360-1369.

24. Claure-Del Granado R, Macedo E, Chertow GM, Soroko S, Himmelfarb J, Ikizler TA, Paganini EP, Mehta RL: Effluent volume in continuous renal replacement therapy overestimates the delivered dose of dialysis. Clin J Am Soc Nephrol 2011, 6:467-475.

25. Lyndon WD, Wille KM, Tolwani AJ: Solute clearance in CRRT: prescribed dose versus actual delivered dose. Nephrol Dial Transplant 2011, 27:952-956.

doi:10.1186/cc11835

Cite this article as: Wald et al.: Optimal Mode of clearance in critically ill patients with Acute Kidney Injury (OMAKI) - a pilot randomized controlled trial of hemofiltration versus hemodialysis: a Canadian Critical Care Trials Group project. Critical Care 2012 16:R205. 